
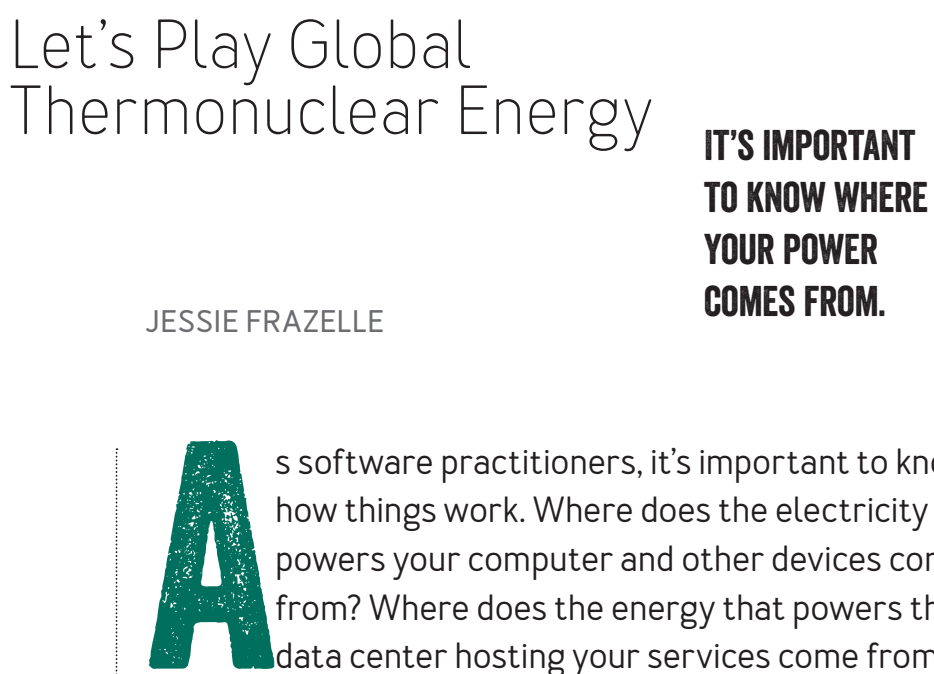

s software practitioners, it's important to know how things work. Where does the electricity that powers your computer and other devices come from? Where does the energy that powers the data center hosting your services come from? Are these efficient sources of energy? Are they good or bad for the environment?

There is a bigger picture involved in the work we do every day. By understanding how aspects of this bigger picture work, we can make sure we are building and using the most efficient systems. As we dive into the physics behind energy, we can learn ways of making longer-lasting batteries to power our devices or find even better means of providing electricity. This article focuses on the latter.

The plasma core of the sun is very hot-about 27 million degrees Fahrenheit [jpl.nasa.gov)-and very dense. The intense mass and heat of the sun's core are what squeezes two hydrogen nuclei in the center of the sun into one helium atom, releasing energy. This process is known as thermonuclear fusion, or more commonly shortened to fusion. During the process, some of the mass of the hydrogen atoms is released as energy, in the form of light. The details of this process are addressed in more detail later. Fusion typically involves two hydrogen nuclei, often deuterium and tritium; but in rare circumstances, in very dense stars four hydrogen nuclei will bond. Fun fact: the 
namesake for helium is the Greek word helios, which means sun. Helium was discovered on the sun during a solar eclipse almost 30 years before the element was found on Earth (cora.nwra.com).

The sun is just a star. Stars share the same process of fusion: converting hydrogen atoms to helium and releasing energy. Larger stars can continue fusion into much heavier elements, until it results in iron. Iron cannot be fused into anything heavier because of the energy required. Once a star creates iron, the next action is usually a supernova, since the star is no longer able to maintain equilibrium. The sun is Earth's main source of energy, but it does not produce the most energy of any star; many other stars produce more energy [courses.lumenlearning.com).

Stars, including the sun, are just one example of fusion. Many scientists are working on making fusion a source of energy to replace the current means of providing power to the grid. Fusion is a promising source of energy for many reasons, which this article covers; it also presents some of the technical details behind fusion energy. And you can't talk about fusion without mentioning fission, so the article touches on this as well. Let's dive in.

\section{BINDING ENERGY AND FUELS}

The example of the stars begins to explain how fusion works. For stars, the fuel for the fusion reaction is hydrogen. To understand nuclear sources of energy, first you need to understand the concept of BE (binding energy]. The BE of a nucleus is equal to the amount of energy required to disassemble it completely into separate protons and neutrons (courses.lumenlearning.com). In 
essence, some nuclei require more energy than others in order to hold them together.

The best fuels for fusion need quite a bit of BE to keep them together. This is in contrast to tightly bound nuclei that do not need much BE to stay together. Combining two hydrogen nuclei that require a lot of BE into a tightly bound helium nucleus is how the fusion reaction occurs in stars. When this happens, the configuration of the nuclei changes, which makes the unneeded BE available. The greater amount of BE that was required for holding the hydrogen nuclei together was no longer required for the tightly bound helium nucleus; therefore, it was released. This is the fundamental interaction behind nuclear fusion energy.

The process of creating energy with fission is a bit converse to the process of fusion. In fission, a heavy, unstable nucleus is split into two lighter nuclei, releasing vast amounts of energy. Fission is used in all nuclear power reactors today. While fusion joins nuclei together to release energy, fission does the opposite, splitting a nucleus into two or more nuclei. For example, a fission reactor takes a heavy uranium nucleus that requires a greater amount of BE and splits it into two tighter-bound, lighter nuclei. Fun fact: The first fission reactor was created in Chicago as a part of the Manhattan Project in 1942 and was named Chicago Pile-1, or CP-1 (energy.gov). Another fun fact: The world's first nuclear submarine, USS Nautilus, used a fission reactor for power. It did not need to be refueled and could remain submerged for months (history.com). 
Any thermonuclear energy process either splits nuclei [fission] or combines nuclei [fusion] to achieve a net gain in energy. Both fission and fusion require starting with nuclei that require a greater amount of $\mathrm{BE}$ and moving toward nuclei that require less $\mathrm{BE}$, releasing the leftover $\mathrm{BE}$ in the process. That leftover energy typically comes in the form of heat and can be used to produce steam in a heat exchanger. The steam then drives a turbine to produce electricity. This is how most fission-based power plants work.

The electric force between charged bodies at rest is known as electrostatic, or Coulomb, force. Any fuel used for fusion would need to overcome the Coulomb force so that it could be fused. This requires a certain amount of energy as input. For any fuel, the energy input required to overcome the Coulomb barrier should be reasonable. Essentially, it takes energy to make energy, but not too much energy such that there is not a good net gain. Most importantly, the energy output from the reaction needs to be net positive; otherwise, it is just a fancy and expensive experiment. In a lot of cases, for example with the sun, there is not enough energy to overcome the Coulomb barrier, and quantum tunneling is required for fusion to work (forbes.com).

The best fuels for fusion require a low amount of energy to induce a reaction. Achieving a reaction needs to be technologically reasonable and economically viable by requiring less energy input than output. The fuel needs to have a high probability of fusion. The nuclei that make up the fuel need to have a greater BE so they will be drawn to a more tightly bound configuration with a minimal amount 
of energy input. Lastly, an ideal fusion fuel has a high energy output that can be converted to electricity.

There are a few options for ideal fusion fuels. A lot of commercial fusion reactors use deuterium as a part of the fuel input. Deuterium, also known as heavy hydrogen or hydrogen-2, is typically denoted by either ${ }^{2} \mathrm{H}$ or $\mathrm{D}$. Hydrogen has two stable isotopes: protium (known as hydrogen-1] and deuterium. Why deuterium? Deuterium fusion generates around 30 times more energy than hydrogen-1 fusion (undsci.berkeley.edu).

The next question is: Deuterium and what? You need two nuclei to make up the fuel. There are three options: deuterium and tritium $(D+T)$; deuterium and deuterium (D + D); and deuterium and helium-3 $\left(\mathrm{D}+{ }^{3} \mathrm{H}\right)$. Each fuel outputs a form of helium and typically something else such as a proton (p), neutron [n], or tritium (T).

One of the main risks for each type of fuel is the probability of Coulomb scattering. This is what occurs when two particles get close to one another and repel each other, flying off in different directions, rather than fusing. If you confine the particles and they scatter, they will bounce off the borders of the confinement back toward each other instead of scattering away infinitely. When all these particles are confined, it creates a plasma. The energy of a plasma, or any substance, is described by its temperature. Temperature is the root mean square of the kinetic energy of every particle in the system. Therefore, the plasma core of the sun at 27 million degrees Fahrenheit has a lot of energy.

Figure 1 shows a table of the most ideal fusion fuels, ranked by their feasibility. The reason for the ranking is first 
FIGURE 1: MOST IDEAL FUSION FUELS

\begin{tabular}{|c|c|c|c|c|}
\hline RANK & FUEL (INPUT) & EXHAUST (OUTPUT) & ENERGY GAIN (MEV) & PEAK REACTIVITY $\left[M^{-3} \mathrm{~S}^{-1}\right]$ \\
\hline 1 & $D+T$ & ${ }^{4} \mathrm{He}+\mathrm{n}$ & 17.6 & $1 \times 10^{-18}$ @ $15 \mathrm{keV}$ \\
\hline 2 & $D+D$ & $\begin{array}{l}\mathrm{T}+\mathrm{p}[50 \%] \\
{ }^{3} \mathrm{He}+\mathrm{n}[50 \%]\end{array}$ & 3.7 & $1 \times 10^{-20}$ (a) $20 \mathrm{keV}$ \\
\hline 3 & $\mathrm{D}+3 \mathrm{H}$ & $3^{4} \mathrm{He}$ & 18.3 & $2 \times 10^{-20}$ @ $50 \mathrm{keV}$ \\
\hline
\end{tabular}

based on the temperature, in keV (kiloelectronvolts). For example, $15 \mathrm{keV}$ is about 175 million degrees $\mathrm{C}$. That's hot! The fuels ranked in positions 2 and 3 require even hotter temperatures, making them not as easy to work with. The required temperature must be practically achievable.

Second, the ranking is based on the energy output. The heat generated from the reaction is used to generate electricity.

\section{COLD FUSION}

As mentioned before, the core of the sun is around 27 million degrees F. For fusion to occur, the fuel typically needs to be very hot. Cold fusion, also called LENR (low energy nuclear reaction], refers to the process of fusion at room temperature. This is compelling because it negates the need for all the energy required to get to a very hot temperature for fusion to occur. This results in a more efficient process that gains more energy to be used for power.

In 1985, as the result of a lab accident, Stanley Pons and Martin Fleischman thought the fusion of deuterium into helium had occurred in one of their electrochemical beakers. The beaker was in a room-temperature lab, so this would have been the first known occurrence of cold fusion. 
If true, the act of performing cold fusion in something as mundane as a lab beaker would be revolutionary, since, as we know from stars, fusion typically takes an incredible amount of heat to be possible. Pons and Fleischman decided to invest their own money into further research of cold fusion as a power source.

After much experimentation, however, they did not gain any more understanding. They could repeat the phenomenon on rare occasions, but there was no explanation why it worked only sometimes, with no path to high-frequency repeatability. The majority of the experiments produced no energy. In 1989, against their better judgment (newenergytimes.com), Pons and Fleishman published their notorious paper: "Electrochemically Induced Nuclear Fusion of Deuterium" [sciencedirect.com].

What happened next has been referred to as the Machiavelli effect in science (amazon.com), meaning many people dismissed the idea of cold fusion. If cold fusion were possible, the high-energy physics community would have been embarrassed and could have lost funding. Cold fusion would have been significantly cheaper than any proposed fusion or fission technology used at that time.

Most people refer to cold fusion as a pathological science-research on it has continued despite a scientific consensus that has established it as false. As shown later in this article, cold fusion does not stand up to the criteria required for fusion energy. That does not explain the phenomenon that Pons and Fleishman uncovered, however. Science optimists find it hard to rule out cold fusion without any explanation as to why the phenomenon 
occurs sometimes; this may explain why some people are still looking into it.

\section{WHY FUSION?}

Fusion is a compelling technology as a source of energy for a number of reasons, including economics, sustainability, and efficiency. Today fission reactors produce an output of 1-1.7 gigawatts of energy. The average cost per kilowatt of electricity is estimated to be similar to that of fission, but it gets less and less expensive with economies of scale liter. org].

With fusion, as opposed to fission, there is no likelihood of a meltdown reaction such as the ones that occurred in Fukushima (world-nuclear.org) and Chernobyl (worldnuclear.org]. This is because fusion requires such a high temperature for the reaction to occur that with any disturbance the plasma would cool within nanoseconds and the reaction would stop.

Fusion does not require or create any materials that could be turned into nuclear weapons, does not emit any toxins such as carbon dioxide into the atmosphere, and does not create any long-lived nuclear waste. The activation of components in a fusion reactor is low enough for the materials to be recycled or reused within 100 years. As stated earlier, the result of fusion is helium. Helium is rather harmless, other than the side effect of a high-pitched voice if inhaled. (This is a joke. Helium is harmless. Scuba divers even use it on long dives as a replacement for nitrogen.]

Fusion fuels are widely available and nearly inexhaustible. For example, deuterium can be distilled from 
all forms of water, and tritium is an output of the fusion of deuterium and deuterium. Fusing atoms together in a controlled way could release nearly four million times more energy than a chemical reaction such as the burning of coal, oil, or gas and four times as much as nuclear fission reactions, at equal mass. Fusion has the potential to provide the baseload energy needed to deliver electricity to cities and industries.

\section{APPROACHES TO FUSION}

There are many different approaches to fusion energy, illustrated in figure 2. This article focuses on the most recent approaches you might have heard of. This section dives into these fusion approaches and describes some of the more modern designs.

The three components required for fusion energy are: plasma density [ $\mathrm{n}$ ), plasma temperature $(\mathrm{T}$ ), and energy confinement $\left(\tau_{\mathrm{E}}\right]$. This is expressed in the mathematical expression:

$$
\mathrm{n} \times \mathrm{T} \times \tau_{\mathrm{E}}
$$

The Lawson criterion [euro-fusion.org] is a powerful way to assess how close a fusion approach is to achieving the necessary conditions. J.D Lawson wrote about this in 1955, which means we have known the criteria for a thermonuclear reactor for decades. Note that cold fusion cannot be modeled with the Lawson criterion.

It uses the three requirements mentioned here and a simple formula:

$$
Q=\text { (fusion energy output) } \div \text { (energy input) }
$$




\section{FIGURE 2: CONCEPTUAL TAXONOMY OF ALTERNATIVE FUSION APPROACHES}

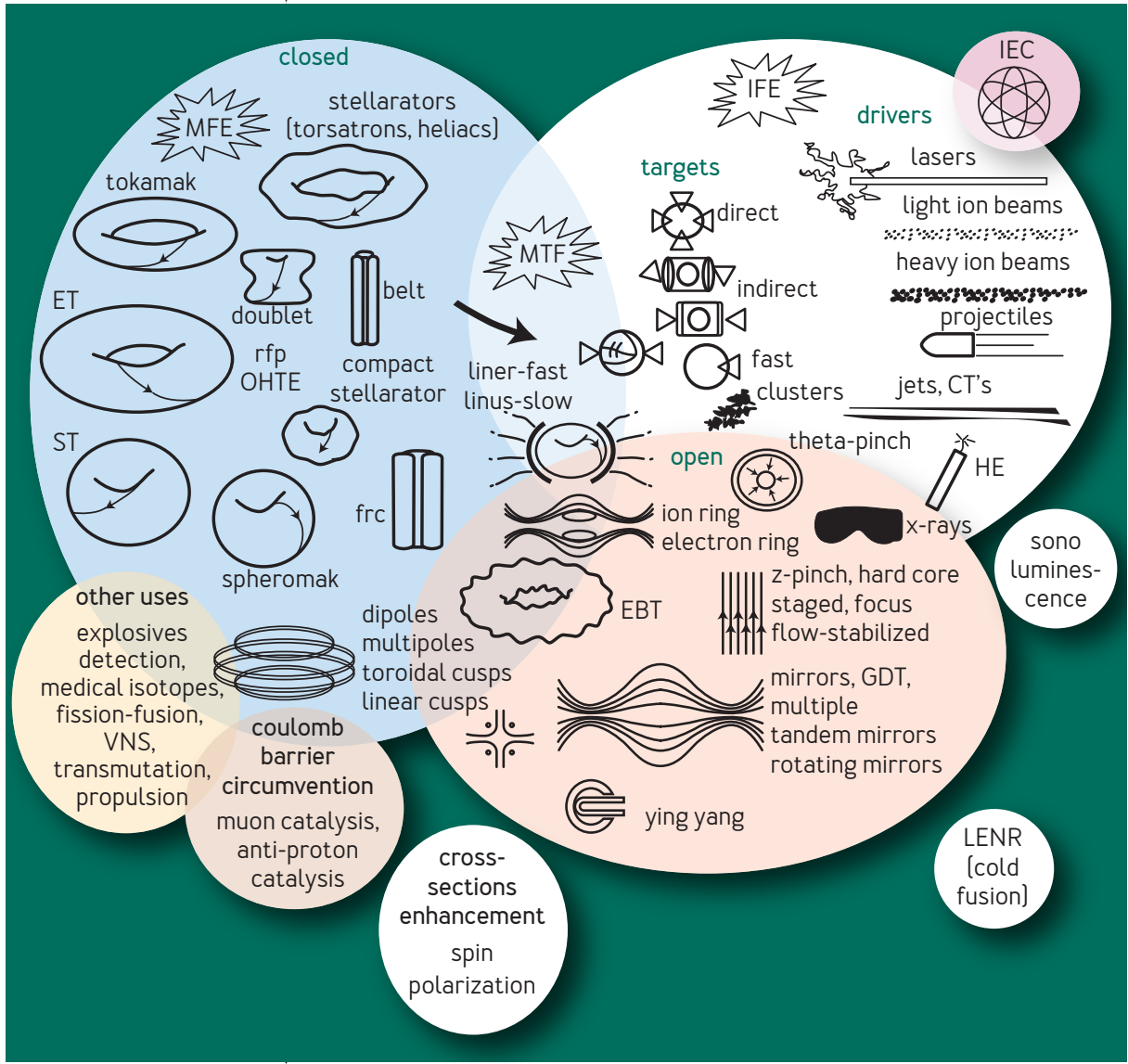

Figure adapted from Woodruff 2004. 10.1007/s10894-004-1869-z

Therefore, to make energy, you need a high Q. Q needs to be greater than 1 to have a gain in energy. When $\mathrm{Q}$ is less than 1 there is energy loss. When $Q$ equals 1 the energy gained is equal to the energy input, so it is neutral; otherwise, you would be consuming energy. A lot of 
concepts fail because of some plasma instability (wikipedia. org); these are the unknown unknowns in the process.

Now that we have a formula for assessing fusion designs, let's take a look at some.

Magnetic-confinement fusion

MCF (magnetic-confinement fusion) features powerful electromagnetic fields that confine and heat plasma inside a donut-shaped reactor called a tokamak. (The name tokamak is derived from a Russian acronym for "toroidal chamber with axial magnetic field.") About 170 devices use this approach, six of which were built at MIT. Many of these technical advances were made possible by computer modeling. The magnetic fields required to confine the plasma are so complex they require a level of precision in manufacturing that did not exist 30 years ago. JET (Joint European Torus) (euro-fusion.org), ITER (iter.org), Commonwealth Fusion Systems [cfs.energy], and Tokamak Energy [tokamakenergy.co.uk] take this approach.

This design has had reactors that have a $\mathrm{Q}$, defined by the Lawson criterion, of around 0.65 (youtube.com]. MCF has the highest $Q$ of any fusion approach to date. Because of the promising early results, the tokamak has become the dominant focus of fusion research; hence, this is why so many MCF devices have been built.

\section{Inertial-confinement fusion}

Lasers! In ICF (inertial-confinement fusion), powerful pulsed laser or ion beams compress a small fuel pellet to extremely high densities. The resulting shock wave heats the plasma before it has time to dissipate. NIF (National 
Ignition Facility] [lasers.llnl.gov) at Lawrence Livermore National Laboratory, OMEGA [sciencedirect.com], Laser Mégajoule (LMJ) Project [sciencedirect.com], First Light Fusion (firstlightfusion.com), and General Atomics (ga.com) take this approach.

The Lawson criterion for NIF gives an estimated Q of 0.1, which is less than 1 , so it is not a net gain in energy.

\section{Magnetized target fusion}

In MTF (magnetized target fusion), also known as MIF (magneto-inertial fusion], magnetic fields confine a lowerdensity plasma, which is then heated and compressed using an inertial-confinement method such as lasers or pistons, similar to inertial-confinement fusion. This concept is a bit like a combination of MCF and ICF. Z-machine (sandia.gov] at Sandia National Laboratory, DPF (Dense Plasma Focus) (ieee.org) at Lawrence Livermore National Laboratory [llnl.gov), ZaP [aa.washington.edu) at the University of Washington, General Fusion [generalfusion.com), HyperJet Fusion (hyperjetfusion.com), and Magneto-Inertial Fusion Technologies (miftec.com) use this approach.

Applying the Lawson criterion to this approach results in a $\mathrm{Q}$ that is less than 0.1. This means the concept, on paper, does not yet have a net gain in energy. The reason why a lot of labs are taking this approach is that the reactor is useful as a high-power X-ray, neutron source, or particle accelerator.

Field-reversed configuration

In FRC [field-reversed configuration], the reactor contains plasma in its own magnetic field by inducing a toroidal 
electric current inside a cylindrical plasma. Compared with the direction of an externally applied magnetic field, the axial field inside the reactor is reversed by eddy currents in the plasma. Helion Energy (helionenergy.com), Princeton Fusion Systems (princetonfusionsystems.com), and TAE Technologies (tae.com) take this approach. TAE Technologies' reactor uses plasma guns to accelerate two plasmas into each other and then heats them with particle beams.

\section{Stellarator}

The stellarator design was one of the original fusion concepts. It features a spiraling ribbon shape that produces high-density plasma. The plasma is symmetrical and more stable than a tokamak, allowing the reactor to run for long periods of time. Wendelstein 7-X lipp.mpg. de] at Max Planck Institute for Plasma Physics uses the stellarator.

The Lawson criterion for this approach gives a Q less than 0.1 .

\section{Liquid fluoride thorium reactor}

Up to this point the article has focused on fusion reactors, touching only briefly on fission. Nuclear power plants today use fission reactors. As already mentioned, during a fission reaction, a heavy nucleus is split into two or more lighter nuclei, releasing energy in the process. Nuclear power plants typically use uranium-235 or plutonium-239 as the fuel for the reactor. Uranium-235 is an isotope of uranium; unlike uranium-238, the predominant and stable isotope, uranium-235 is fissile, meaning it can sustain a 
fission chain reaction. The most common type of fission reactor is the LWR (light-water reactor), which uses normal water, versus heavy water, as the coolant and neutron moderator.

A LFTR [liquid fluoride thorium reactor), pronounced lifter, uses thorium along with a fluoride-based, molten, liquid salt for the reactor fuel. This design is a type of molten salt reactor, in which the primary coolant andlor fuel are a molten salt mixture. In the case of a LFTR, both the coolant and the fuel are a molten salt mixture. What is unique about molten salt reactors is that they operate close to atmospheric pressure, as opposed to the 75-150 times the atmospheric pressure of typical LWR operations. Operating at atmospheric pressure helps prevent accidents (hal.in2p3.fr].

The first LFTR concept was investigated at Oak Ridge National Laboratory in the 1960s, but it did not use thorium. Many countries are looking into LFTR today. One startup, Flibe Energy [flibe-energy.com], is working on building a LFTR.

What advantages does a LFTR have over existing fission reactors? The main advantage is safety. As just mentioned, operating near atmospheric pressure helps prevent the explosions that can occur at very high pressure. Thorium and LFTR waste byproducts are hard to turn into nuclear weapons and have a much shorter half-life than traditional LWRs (scidev.net]. You can safely hold thorium in your hands, which you cannot do with uranium or plutonium. In fact, using thorium versus uranium as a fuel would mean that the prevalence of enriched uranium, one of the most commonly used materials for nuclear weapons, would be 
much smaller (semanticscholar.org).

Thorium is in abundance in the earth's crust, even more than tin, mercury, or silver. Using thorium has a huge upside in that it allows for thermal breeder reactors. This means the thorium fuel can breed fissile uranium, which can in turn be reprocessed in the reactor to create more energy. This allows the nuclear fuel resources on Earth to be extended by two orders of magnitude. In typical fission reactors, uranium needs to be enriched before it can be used as a fuel. Conventional LWRs consume less than 1 percent of the mined uranium, leaving the rest as waste. A LFTR with reprocessing can consume about 99 percent of its thorium fuel, and the thorium fuel is 100 percent natural with no need for enrichment.

What about economics? The vast natural abundance of thorium combined with the efficiency of the fuel versus typical fissile reactors suggest the economic advantages would be straightforward. In 2014, however, researchers at the University of Chicago published a paper stating otherwise. They acknowledged there was a difference in cost, but argued that it was not sufficient to justify someone building a reactor since it would take a large amount of knowledge, expertise, and time to accomplish [semanticscholar.org]. I want to believe it was fear and a lack of optimism that led to this conclusion, since the longterm economic and safety advantages would be a huge achievement.

\section{CONCLUSION}

By now you should have a greater understanding of the fusion and fission reactors that make up thermonuclear 
energy solutions. By starting from first principles, this article has shown what occurs to nuclei in nuclear reactions. For us to grow and progress as a civilization, we need more investment in providing electricity to the world through clean, safe, and efficient processes. Thermonuclear energy is a huge step forward.

This article has mostly focused on the use cases around grid-scale reactors. There are lots of other use cases for nuclear energy: atomic batteries, nuclear engines for rockets (wikipedia.org], space rovers, submarines [upi. com], and aircraft [wikipedia.org]. It's hard to see a future without some sort of thermonuclear energy powering all sorts of things around us. So, to slightly modify a line from the movie WarGames, "Let's play global thermonuclear energy!"

Acknowledgements

Thank you to Thomas Eckert, James Casey, and Jordan Noone for providing feedback on this article.

Jessie Frazelle is the cofounder and chief product officer of the Oxide Computer Company. Before that, she worked on various parts of Linux, including containers, as well as the Go programming language.

Copyright $(2020$ held by ownerlauthor. Publication rights licensed to ACM. 